\title{
Fault diagnosis of plunger pump in truck crane based on relevance vector machine with particle swarm optimization algorithm
}

\author{
Wenliao $\mathrm{Du}^{\mathrm{a}, \mathrm{b}, *}$, Ansheng $\mathrm{Li}^{\mathrm{b}}$, Pengfei Ye ${ }^{\mathrm{a}}$ and Chengliang Liu ${ }^{\mathrm{a}}$ \\ ${ }^{a}$ State Key Laboratory of Mechanical System and Vibration, Shanghai Jiao Tong University, Shanghai, China \\ ${ }^{\mathrm{b}}$ School of Mechanical and Electronic Engineering, Zhengzhou University of Light Industry, Zhengzhou, Henan, \\ China
}

Received 14 February 2012

Revised 9 August 2012

Accepted 22 October 2012

\begin{abstract}
Promptly and accurately dealing with the equipment breakdown is very important in terms of enhancing reliability and decreasing downtime. A novel fault diagnosis method PSO-RVM based on relevance vector machines (RVM) with particle swarm optimization (PSO) algorithm for plunger pump in truck crane is proposed. The particle swarm optimization algorithm is utilized to determine the kernel width parameter of the kernel function in RVM, and the five two-class RVMs with binary tree architecture are trained to recognize the condition of mechanism. The proposed method is employed in the diagnosis of plunger pump in truck crane. The six states, including normal state, bearing inner race fault, bearing roller fault, plunger wear fault, thrust plate wear fault, and swash plate wear fault, are used to test the classification performance of the proposed PSO-RVM model, which compared with the classical models, such as back-propagation artificial neural network (BP-ANN), ant colony optimization artificial neural network (ANT-ANN), RVM, and support vector machines with particle swarm optimization (PSOSVM), respectively. The experimental results show that the PSO-RVM is superior to the first three classical models, and has a comparative performance to the PSO-SVM, the corresponding diagnostic accuracy achieving as high as $99.17 \%$ and $99.58 \%$, respectively. But the number of relevance vectors is far fewer than that of support vectors, and the former is about $1 / 12-1 / 3$ of the latter, which indicates that the proposed PSO-RVM model is more suitable for applications that require low complexity and real-time monitoring.
\end{abstract}

Keywords: Plunger pump, truck crane, fault diagnosis, particle swarm optimization, relevance vector machines

\section{Introduction}

With the rapid growth in modernization program and the constant expansion of resource exploitation, the truck cranes are facing a heavy demand on their services, and the maximum lifting weight has exceeded 1000 tons. The much higher request on reliability and safety is bring up for the machine, and promptly and accurately dealing with the equipment breakdown is very helpful in terms of enhancing reliability and decreasing downtime. The plunger pump constitutes the key part of truck crane, and the quality of the pump affects the performance of the hydraulic system, even the whole equipment, directly. So, it is very important to be able to realize on line monitoring and diagnose the faults as early as possible. To this end, a considerable number of researches have focused on this

* Corresponding author: W. Du, State Key Laboratory of Mechanical System and Vibration, Shanghai Jiao Tong University, Shanghai 200240, China. Tel.: +86 213420 4350; Fax: +86 213420 6529; E-mail: dwenliao@zzuli.edu.cn. 
field. Chen et al. [1] utilized the vibration signal's energy obtained by the continuous wavelet transform, and Wang et al. [2] employed the Hilbert transform and the genetic algorithm to obtain the characteristic frequencies of the vibration signals, which are used to determine the size and the position of the crack in plunger of water hydraulic motor. Zhao et al. [3] proposed a method based on intermittent chaos and sliding window symbol sequence statistics for loose shoes early fault diagnosis of hydraulic pump. Mollazade et al. [4] presented a fuzzy inference system in combination with decision trees based method for gear hydraulic pump. Furthermore, Chen et al. [5] studied the fault degradation assessment of the water hydraulic motor based on wavelet packet analysis and Kolmogorov-Smirnov test.

Essentially, fault diagnosis can be regarded as a pattern recognition problem. Some intelligent classification technologies, such as artificial neural networks ANNs [6,7] and SVMs [8] have been successfully applied to the fault diagnosis of rotating machinery. In real applications, the SVMs seem to prevail in the field of intelligence fault diagnosis for their favorable generalization ability [9]. But the SVM model itself has some inherent limitations. First, the SVM classifier is a typical point estimation method instead of a probabilistic estimation one. Second, it is necessary estimate the error/margin trade off parameter ' $\mathrm{C}$ ' by cross validation procedure, and the kernel function must satisfy Mercer's condition. Finally, the number of support vectors increases linearly with the size of training sample set, which requires much higher computational complexity when classifying a very large data set. Solving these problems is very critical for the real time monitoring and one line fault diagnosis.

The RVM model proposed by Tipping [10] has been successfully used in bearing fault diagnosis [11], gear fault diagnosis [12], electronic nose data analysis [13], fMRI signal processing [14], and so on. In addition, Caesarendra et al. assessed the bearing degradation with RVM [15]. Compared with SVM, RVM is based on the Bayesian estimation and offers a probabilistic prediction of the result. Moreover, it can choose arbitrary kernel functions. In general, the most popular kernel used in RVM is the radial basis function (RBF) kernel. Thus, only the kernel width parameter needs to be estimated. More importantly, RVM classifier utilizes significantly fewer relevance vectors, while providing a similar classification performance as compared with SVM approach. This feature makes the RVM classifier more suitable for the application that requires low complexity and real time diagnosis. PSO motivated by social behavior of bird flocking is an example of a modern search heuristics optimization technology [16]. In the PSO method, the particles are "flying" in the solution space, remembering the individual optimal position and the global optimal position, and changing their positions and communicating to each other, until the optimal solution is found. This optimization approach is easy to implement and has a good robustness. It has been successfully applied to solve multi-dimensional optimization problem in artificial neural network [17] and support vector machine [18]. In this study, the proposed PSO-RVM model is utilized to diagnosis the plunger pump in truck crane, in which PSO is used to determine the kernel width parameter.

This paper is organized as follows: Section 2 introduces the RVM classifier. Parameter optimization of RVM based on PSO is realized in Section 3. The diagnostic PSO-RVM model for plunger pump in truck crane is presented in Section 4. Section 5 testifies the performance of the proposed model with the real application. The performance comparison between the PSO-RVM method and the classical intelligent classifiers is also implemented. Finally, the conclusion is provided in Section 6.

\section{RVM classifier}

The original RVM was derived on binary classification problem where it was desired to predict the posterior probability of membership of these classes for the input data. Given a training data set $\left\{x_{i}, t_{i}\right\}_{i=1}^{N}$, here, $x_{i}$ and $t_{i} \in\{1,0\}$ denote the input vector and the classification label, respectively, and $N$ represents the number of training samples. For a new input vector $x$, the object is to seek an enough accuracy prediction of label $t$. Commonly, this prediction can be reached based on a sparse model defined over the input space in the form of

$$
y(x)=\sum_{m=1}^{M} \omega_{m} \phi_{m}(x)
$$


where, $M$ corresponds to the number of training samples whose weight $\omega$ is not 0 in all $N$ training samples. As only two values ( 0 and 1$)$ are possible for $t$, a Bernoulli distribution can be adopted for $P(t \mid x)$. Apply the logistic sigmoid link function to link random and systematic components, the likelihood is written as

$$
P(t \mid \omega)=\prod_{n=1}^{N} \sigma\left\{y\left(x_{n} ; \omega\right)\right\}^{t_{n}}\left[1-\sigma\left\{y\left(x_{n} ; \omega\right)\right\}\right]^{1-t_{n}}
$$

This function is complemented by a prior over the weight parameters with hyper parameters $\alpha=\left(\alpha_{1}, \ldots, \alpha_{M}\right)$ in the form of

$$
P(\omega \mid \alpha)=(2 \pi)^{-M / 2} \prod_{m=1}^{M} \sqrt{\alpha_{m}} \exp \left(-\frac{\alpha_{m} \omega_{m}^{2}}{2}\right) .
$$

The hyper parameters are used to control the strength of the prior over the corresponding weight. So, the prior satisfies Gaussian, but is conditioned on $\alpha$. For a given $\alpha$, the posterior weight distribution can be obtained by the Bayes rule

$$
P(\omega \mid t, \alpha)=\frac{P(t \mid \omega) P(\omega \mid \alpha)}{P(t \mid \alpha)}
$$

The weights can not be obtained analytically, so the Laplace method is adopted in the following way.

1) As $P(\omega \mid t, \alpha) \propto P(t \mid \omega) P(\omega \mid \alpha)$, maximizing the posterior is equivalent to maximizing the followed formula on $\omega$

$$
\log \{P(t \mid \omega) P(\omega \mid \alpha)\}=\sum_{n=1}^{N}\left[t_{n} \log y_{n}+\left(1-t_{n}\right) \log \left(1-y_{n}\right)\right]-\frac{1}{2} \omega^{T} A \omega
$$

where, $y_{n}=\sigma\left\{y\left(x_{n} ; \omega\right)\right\}$, and $A=\operatorname{diag}\left(\alpha_{1}, \ldots, \alpha_{M}\right)$. Eq. (5) is a penalized logistic log-likelihood function and requires iterative maximization. Usually, the second-order Newton method by adapting the efficient "iteratively reweighted least-squares" algorithm is employed to find $\omega_{M P}$.

2) Laplace method is a quadratic approximation to the log-posterior around $\omega_{M P}$. The Eq. (5) is differentiated twice to obtain the Hessian in the form of

$$
\left.\nabla \omega \nabla \omega \log P(\omega \mid t, \alpha)\right|_{\omega_{M P}}=-\left(\Phi^{T} B \Phi+A\right)
$$

where, $B=\operatorname{diag}\left(\beta_{1}, \ldots, \beta_{N}\right)$ is a diagonal matrix with $\beta_{n}=\sigma\left\{y\left(x_{n}\right)\right\}\left[1-\sigma\left(x_{n}\right)\right]$, and $\Phi=\left[\phi_{1}, \ldots, \phi_{M}\right]$ is a $N \times M$ 'design' matrix whose columns comprise the complete set of $M$ 'basis vectors'. Then, this result is negated and inverted to give the covariance $\Sigma=\left(\Phi^{T} B \Phi+A\right)^{-1}$ for a Gaussian approximation to the posterior over weights centered at $\omega_{M P}$. So, the classification problem is equivalent to locally linearised $\omega_{M P}=\sum \Phi^{T} B \hat{t}$ around $\omega_{M P}$, where $\hat{t}=\Phi \omega_{M P}+B^{-1}(t-y)$.

3) Update the hyper parameters with

$$
\alpha_{i}^{\text {new }}=\frac{\lambda_{i}}{\omega_{i}^{2}}
$$

where, $\omega_{i}$ is the $i$ th element of $\omega_{M P}, \lambda_{i}=1-\alpha_{i} \Sigma_{i i}$, and $\Sigma_{i i}$ is the $i$ th diagonal element of the covariance. During the optimization process, most of $\alpha_{i}$ will have large values, and the corresponding $\omega_{i}$ is close to 0 , so the scarcity is realized.

4) The optimization process continues, until the maximum change of hyper parameters $\alpha$ is below a certain sufficiently small threshold in two successive iterations, or the preset maximum iteration number is reached. All of the input vectors with non zero weight are denoted as relevance vectors. 


\section{Parameter optimization of RVM based on PSO}

In this study, the RBF kernel is selected for RVM. Before training the RVM model, the kernel width parameter $\gamma$ should be predetermined, which has great impact on the classification accuracy. The PSO is applied to select the proper kernel parameter. PSO uses a population (called swarm) of individuals (called particles) that are updated from iteration to iteration. The position of each individual represents a possible solution, and the optimal solution is searched by continual updating the velocity and position. Each particle moves in the direction determined by its previously best position and its best global position. The velocity and position are updated according to the following equations

$$
\begin{aligned}
& v_{i}(t+1)=\omega_{i n} \cdot v_{i}(t)+c_{1} \cdot \operatorname{rand}_{1} \cdot\left(\text { pbest }_{i}(t)-p_{i}(t)\right)+c_{2} \cdot \operatorname{rand}_{2} \cdot\left(\text { gbest }_{i}(t)-p_{i}(t)\right) \\
& p_{i}(t+1)=p_{i}(t)+\beta \cdot v_{i}(t+1)
\end{aligned}
$$

here, $v_{i}$ and $p_{i}$ present the velocity and position of the particle $i$, respectively; pbest $_{i}$ is the best position of particle $i$, and gbest $_{i}$ is the global best position of the swarm; $c_{1}$ and $c_{2}$ are two positive acceleration constants regulating the relative velocities, and they are usually set to 2 ; rand $_{1}$ and rand $_{2}$ are random variables in the range $[0,1] ; \beta$ is a constraint factor used to control the velocity weight, which is usually set to $1 ; \omega_{i n}$ is the inertia weight, which is used to balance the capabilities of global exploration and local exploitation, determined by $\omega_{\text {in }}=\omega_{\max }-\left(\omega_{\max }-\right.$ $\left.\omega_{\min }\right) \cdot t / T$, where $\omega_{\max }$ is the initial weight, $\omega_{\min }$ is the final weight, and $T$ is the maximum number of iterations.

The RVM is trained with the selected kernel parameter, and the objective is to get a most excellent generalization performance of the classification model. The performance is weighted with the fitness function and the problem is treated as an optimization issue. In the training process, the two-fold cross validation is used to evaluate the fitness. First, the training data set is randomly divided into 2 mutually exclusive subsets, $S_{1}$ and $S_{2}$, with approximately equal size. Then, $S_{1}$ is used as the training set and $S_{2}$ is used as the validation set. Finally, $S_{2}$ is used as the training set and $S_{1}$ is used as the validation set. The fitness function can be defined as follows

$$
\text { Fitness }=\frac{1}{2} \sum_{j=1}^{2} \frac{y_{f}(j)}{y_{f}(j)+y_{c}(j)}
$$

where, $y_{f}(j)$ and $y_{c}(j)$ represent the number of false and true classifications in the $j$ th validation set, respectively. The optimization objective is minimizing the fitness value. Figure 1 is the flow chart of optimizing the RVM parameters with PSO, which is represented as follows

1) Initialize the swarm size, maximum of iterations and the velocity and position for each particle. During the optimization process, the velocity and the position are restricted to $\left[-v_{\min },+v_{\max }\right]$ and $\left[-p_{\min },+p_{\max }\right]$, respectively.

2) Train the RVM model, and evaluate the fitness with two-fold cross validation method.

3) Calculate the best position of individual and the global position of the swarm according to the fitness evaluation results.

4) Update the velocity and position of each particle by Eqs (8) and (9), respectively.

5) Repeat the procedures from step (2) to (4) until the stop condition is satisfied. The stop condition is that the fitness value is below a given threshold or the iteration number reaches a given max value.

\section{Diagnostic method for plunger pump in truck crane based on PSO-RVM}

As shown in Fig. 2, the normal state and various fault types data of plunger pump in truck crane are used to verify the proposed method. The features are firstly extracted from the acquired running data. The features and the corresponding labels are utilized to train the RVM model. Then, the test samples are employed to verify the trained model. Before training, the PSO method is used to optimize the parameter of RBF kernel. The classification accuracy, which is represented by the ratio of the number of correct classifications and the number of total test samples, is utilized to evaluate the performance of the model. 


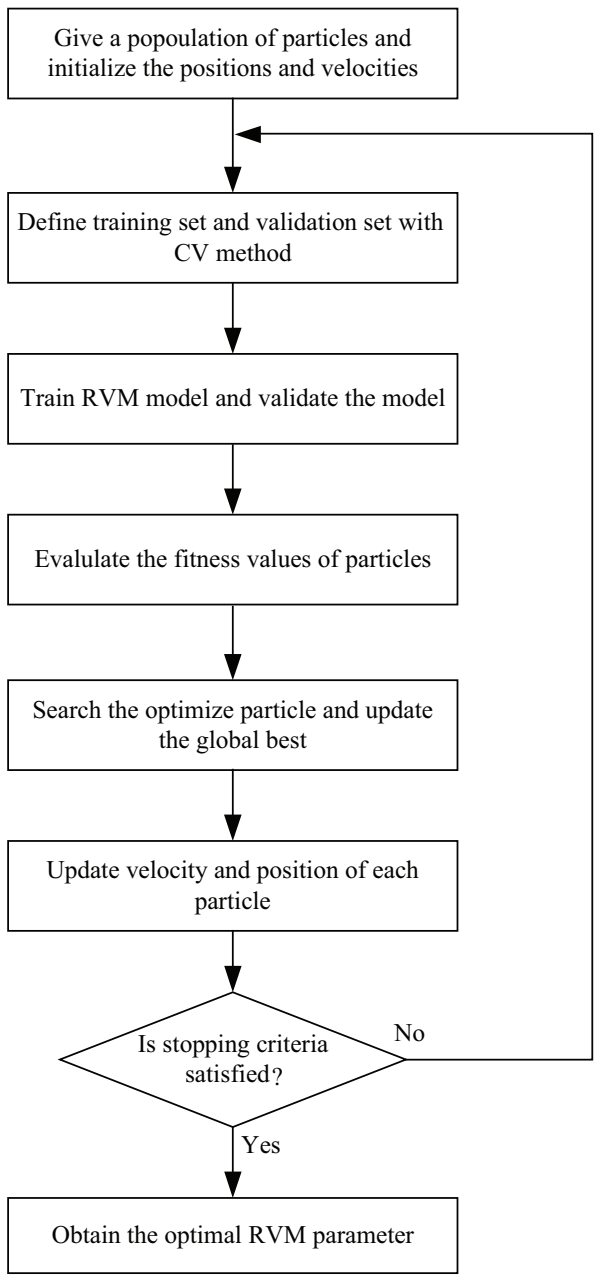

Fig. 1. Process of optimizing the RVM parameters with PSO.

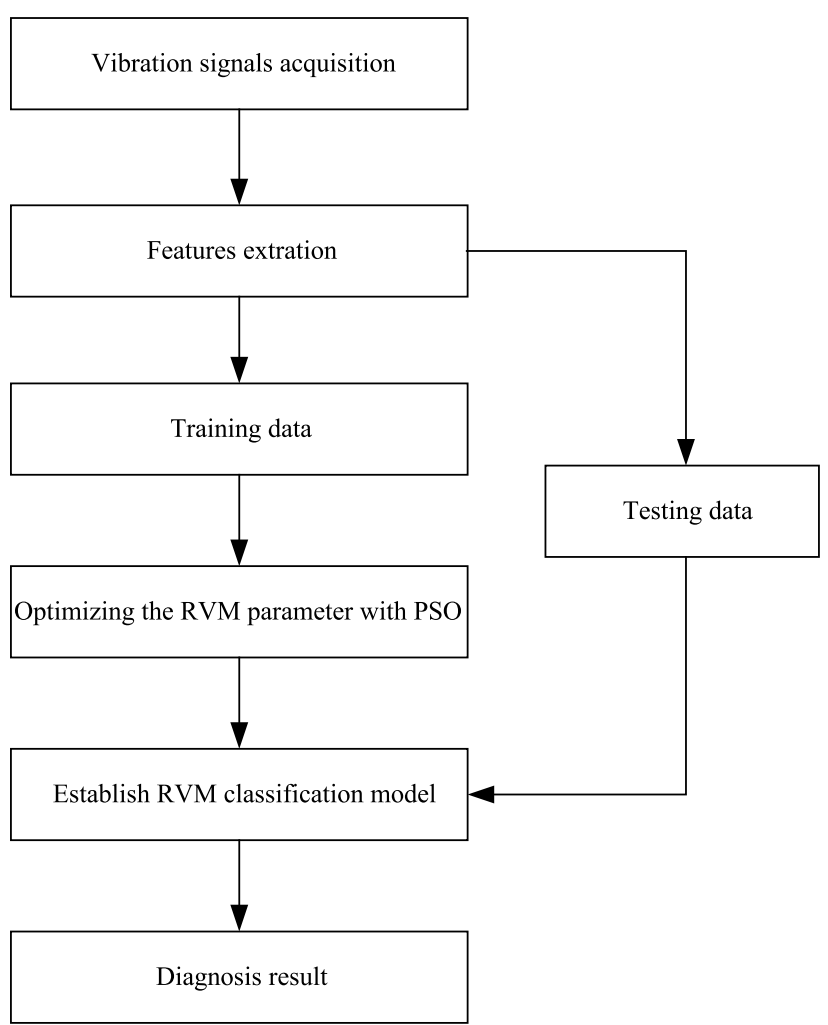

Fig. 2. Diagnostic model of plunger pump based on PSO-RVM classifiers.

\subsection{Feature extraction}

The wavelet packet transform method possesses perfect local property in both time domain and frequency space, and it is widely used in the region of machinery fault diagnosis. In the present work, the wavelet packet energy is extracted to represent the condition of mechanical equipment. The 'daubechies 5' (Db5) wavelet function is employed to decompose the original sample data. The wavelet packet energy is defined as $E_{j, n}=\sum_{k=1}^{N_{j}}\left|c_{j, k}^{n}\right|^{2}$, where, $c_{j, k}^{n}$ denotes the coefficients of the $n$th bins in the $j$ th level of the wavelet packets, $N_{j}$ is the number of wavelet coefficients of each bin in the $j$ th level. So, the total wavelet packet energy of the $j$ th level can be calculated as $E=\sum_{n=1}^{2^{j}} E_{j, n}$. Then, the ratio of the wavelet packet energy can be obtained by $\tilde{E}_{j, n}=E_{j, n} / E$. These ratios of the lowest level depict the energy distribution of the original signal, and the feature vector can be represented as $\vec{F}=\left[\tilde{E}_{j, 1}, \tilde{E}_{j, 2}, \ldots, \tilde{E}_{j, 2^{j}}\right]$.

\subsection{Fault diagnosis model based on two-class PSO-RVM}

The original RVM model is a typical two-class classifier. However, for the plunger pump of truck crane, the ordinary fault forms include bearing inner race fault, bearing roller fault, plunger wear fault, thrust plate wear fault, and swash plate wear fault, and so on. Further more, the normal state can be regarded as an especial fault type. So, this is a multi-class problem. In practical work, in addition to the pointed five fault categories, there can be 


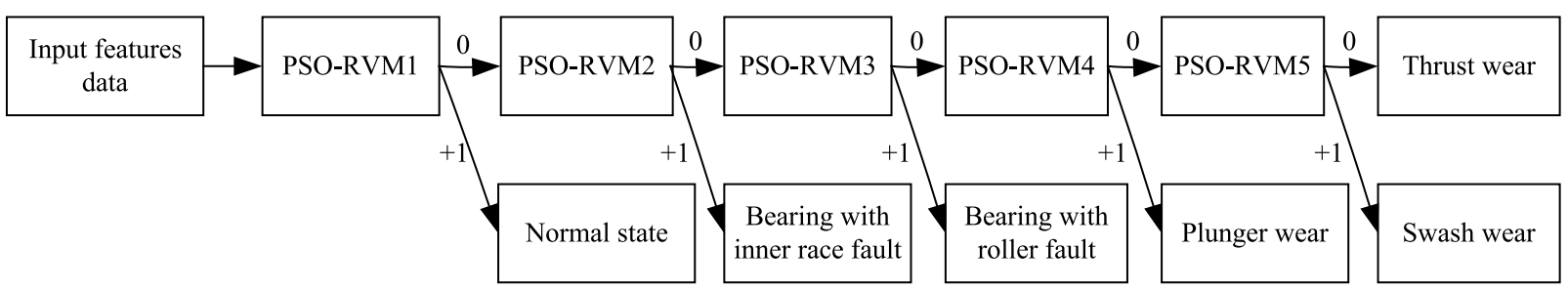

Fig. 3. Diagnosis process of PSO-RVM classifiers.

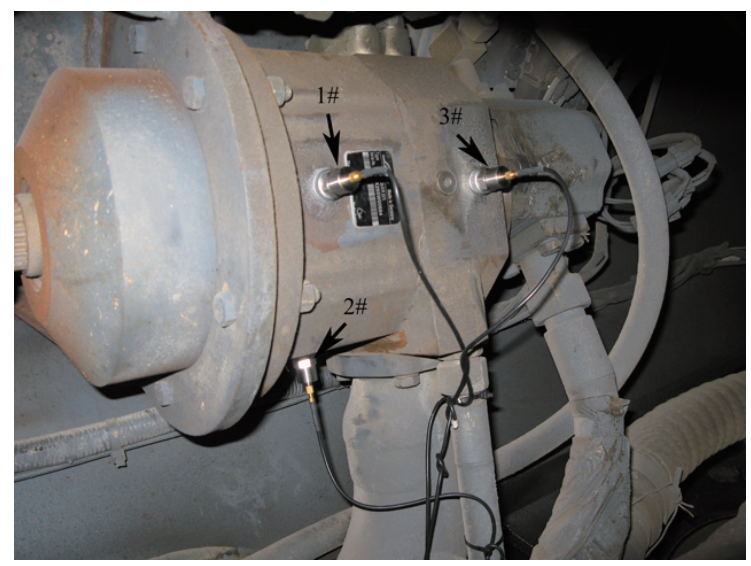

(a)

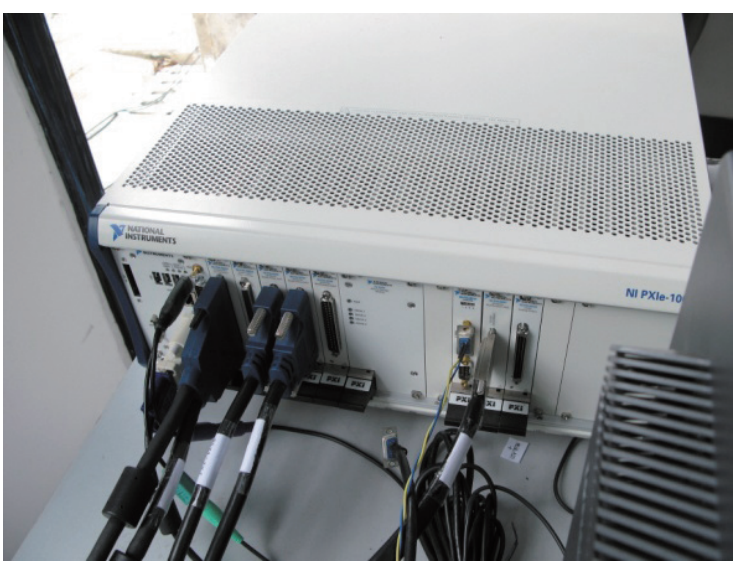

(b)

Fig. 4. Plunger pump test rig. (a) The pump and sensor placement illustration, (b) The acquisition device of vibration signal.

other unknown fault patterns. In such case, the novelty detection approaches, such as SVDD method, immunology principles based method, or other statistic methods, should be integrated into the fault diagnosis model, undoubtedly introducing some complexities. Under the certain circumstance in this manuscript, in order to simplify the question, we only consider these six categories mentioned above. As shown in Fig. 3, several two-class RVMs with binary tree architecture are trained to recognize the condition of mechanism. For a case of $k$ classes, only $k-1$ two-class RVM classifiers are needed. For the present plunger pump, the diagnosis model comprises five two-class RVM classifiers. With all training samples of the six states, PSO-RVM1 is trained to separate the normal state from fault states. When the input is a sample representing normal state, the output is set to 1 , otherwise, the output is set to 0. PSO-RVM2 is trained to separate bearing inner race fault from the other four fault states. When input of PSO-RVM2 is a sample representing bearing inner race fault, the output of PSO-RVM2 is set to 1, otherwise, the output is set to 0 . Utilize the samples with bearing roller fault, plunger wear fault, thrust plate wear fault, and swash plate wear fault to train PSO-RVM3. When input of PSO-RVM3 is a sample representing bearing roller race fault, the output of PSO-RVM3 is set to 1 , otherwise, the output is set to 0. Similarly, Utilize the samples with plunger wear fault, thrust plate wear fault, and swash plate wear fault to train PSO-RVM4. When input of PSO-RVM4 is a sample representing plunger wear fault, the output of PSO-RVM4 is set to 1, otherwise, the output is set to 0. PSO-RVM5 is trained to separate thrust plate wear fault from swash plate wear fault. When input of PSO-RVM5 is a sample representing swash wear fault, the output of PSO-RVM5 is set to 1, otherwise, the output is set to 0 .

\section{Application to plunger pump fault diagnosis}

\subsection{Experimental equipment}

As shown in Fig. 4, the plunger pump is seated on the truck crane. The pump type is Linde HPR130-01R 2576, which has nine plungers. The accelerometer is CA-YD-186, and the range of frequency and sensitivity is $0.1-6 \mathrm{kHz}$ 

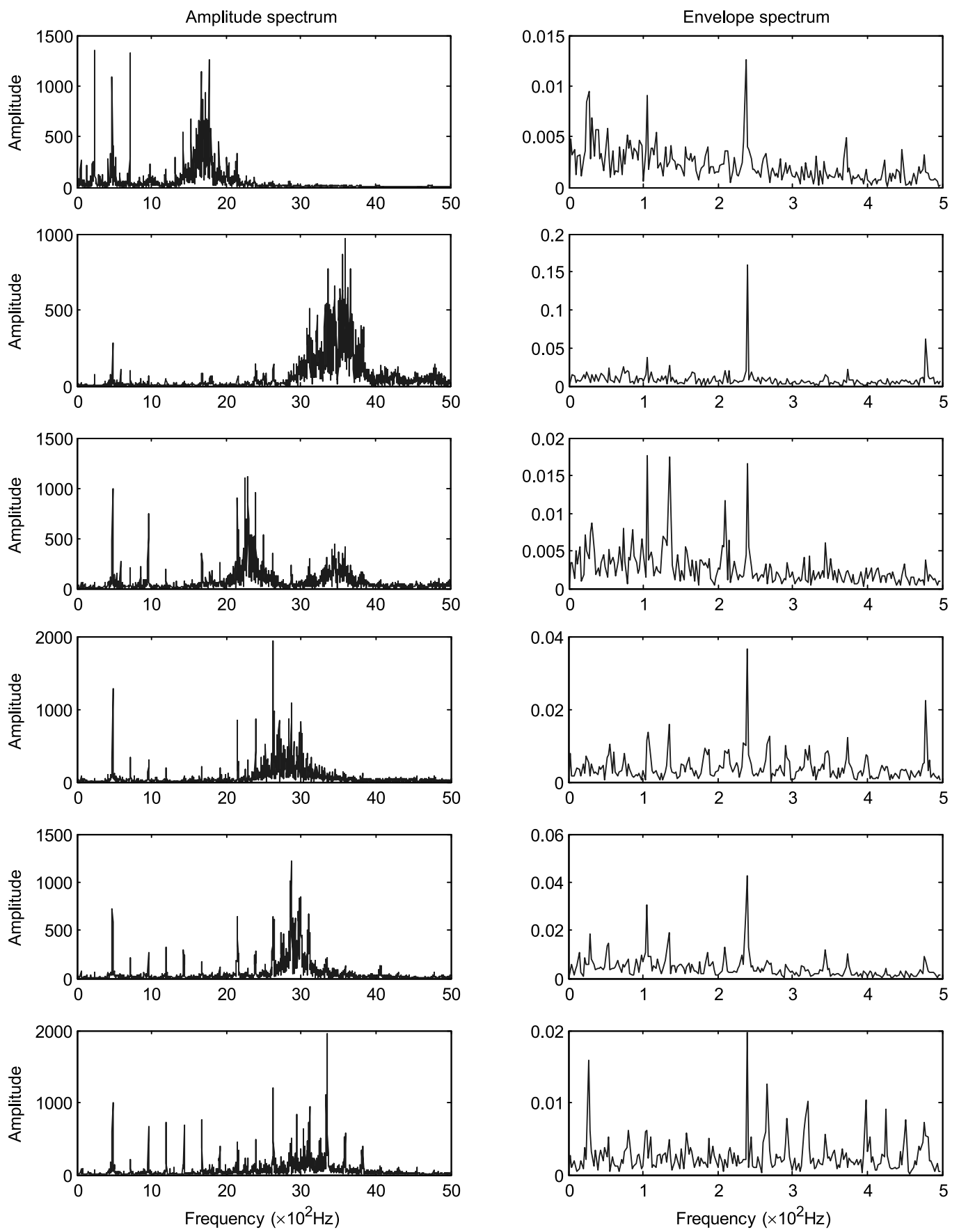

Fig. 5. Amplitude spectrums (left row) and envelope spectrums (right row) of the vibration signal with different fault categories. The panels from up to down correspond to norm state, bearing inner race fault, bearing roller fault, plunger wear fault, swash plate wear fault, thrust plate wear fault, respectively.

and $50 \mathrm{mv} / \mathrm{g}$, respectively. Three accelerometers mounted on the pump surface are used to obtain the vibration signal. The accelerometers are located in the horizontal direction of the bearing (1\#), the vertical direction of the bearing (2\#), and the horizontal direction of thrust plate (3\#), respectively. The digital collecting equipment consists of NI9233 data collection card and software, which has a frequency variable anti-alias filter, and the highest sampling 

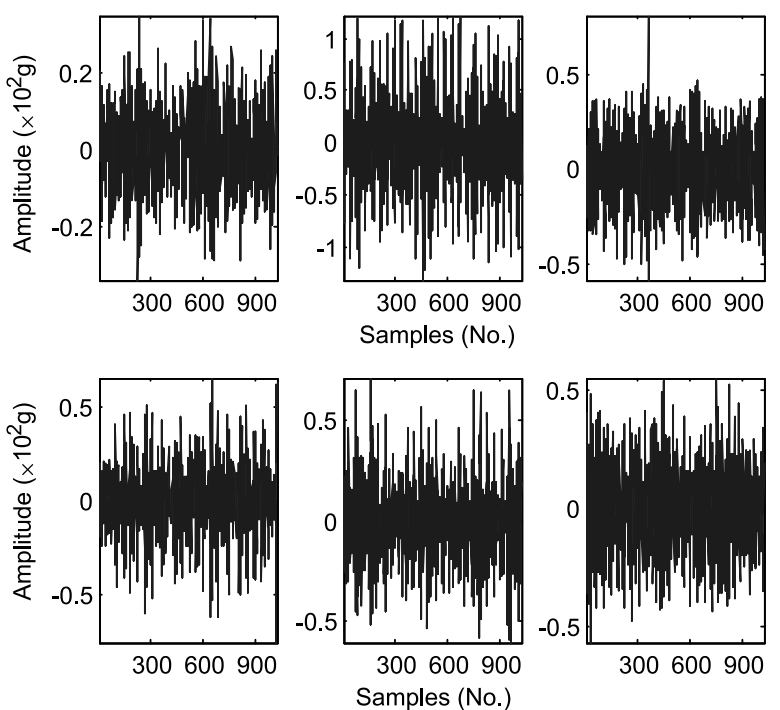

Fig. 6. Waveform of the original vibration signals. Top row, from left to right: normal state, bearing inner race fault, and bearing roller fault. Bottom row, from left to right: plunger wear fault, thrust plate wear fault, and swash plate wear fault.
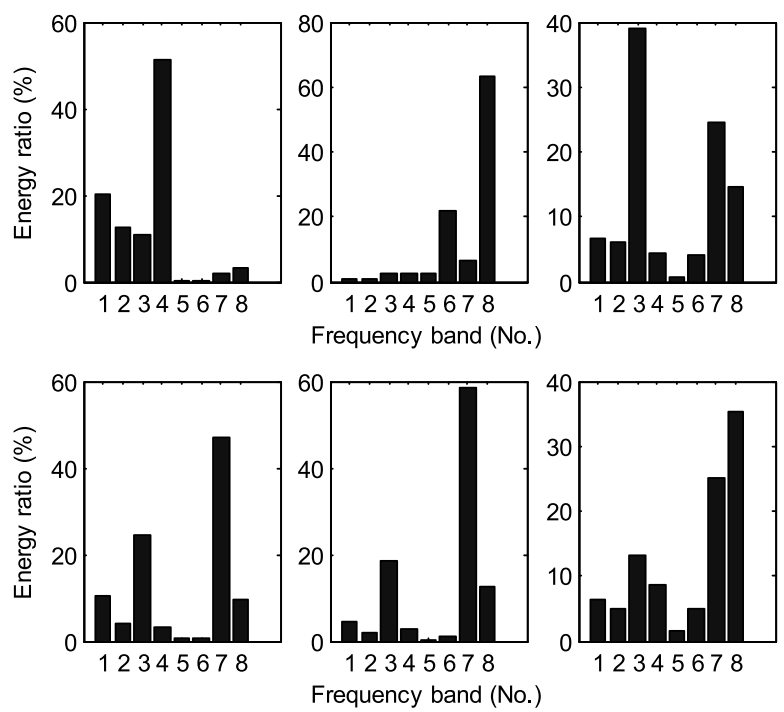

Fig. 7. Eight wavelet packet energy ratios of the typical vibration signals. Top row, from left to right: normal state, bearing inner race fault, and bearing roller fault. Bottom row, from left to right: plunger wear fault, thrust plate wear fault, and swash plate wear fault.

frequency is $50 \mathrm{kHz}$. The rotating speed of the motor is about $1600 \mathrm{r} / \mathrm{min}$, the liquid pressure is $6 \mathrm{MPa}$, and the vibration signals are collected at a sampling rate of $10 \mathrm{kHz}$. For the six states, including normal state, bearing inner race fault, bearing roller fault, plunger wear fault, thrust plate wear fault, and swash plate wear fault, each state is collected about 200000 points. The bearing faults are simulated by cutting a groove with $0.2 \mathrm{~mm}$ depth and $0.3 \mathrm{~mm}$ width on the inner race and the roller surface, and the samples with the plunger wear fault, the thrust plate wear fault, and the swash plate wear fault are provided by a manufactures of truck crane. By the analysis and comparison, the acquired signals of accelerometer $1 \#$ are selected as the input of the diagnosis model.

One of the most popular fault analysis methods today is based on spectrum analysis [19-21]. Under the prior knowledge of the components presented, by the amplitude spectrum or the envelope spectrum, if the fault character frequency is distinct, it can be assessed that the corresponding fault may be immersed. In addition, if the signal is contaminated by noise, the character frequency is not easy to be extracted, and some preconditioning technologies should be adopt to enhance the signal. In the paper [19], the optimal Morlet wavelet filter and autocorrelation enhancement technologies are used before the envelope spectrum is performed. In the recent paper [20], the cyclic wiener filter method is employed to improve the weak fault feature before envelope spectrum analysis. But these technologies require the priori knowledge of the fault [21]. For the pump presented in this manuscript, some fault character frequencies can be calculated conveniently. For example, the bearing character frequencies of the inner race fault and the ball fault can be obtained from the kinematics relation. Furthermore, because there are two impacts between the plunger and the slipper in a period of rotation of the cylinder, the character frequency of plunger wear fault is two times of the axis frequency. But, for the thrust plate wear fault and swash plate wear fault, the character frequencies have not a specific definition, which need further investigation. In Fig. 5, the amplitude spectrum and the envelope spectrum of the pump are provided for the six conditions. Due to the absence of the prior knowledge of fault frequencies, the band pass filter is not used. For the current spectrum chart, though we can observe the frequency of $239 \mathrm{~Hz}$ (according to the nine times of axis frequency) is remarkable in each spectrum chart; the frequency of $105 \mathrm{~Hz}$ (according to the 4 times of axis frequency) is apparent for the normal state, bearing roller fault, and the swash plate wear fault; the axis frequency $(27 \mathrm{~Hz})$ is apparent for the normal state and the thrust plate wear fault; other prominent frequencies for some states have no distinct meaning. We can not determine the fault category directly. Especially, from this figure, the spectrums corresponding to the plunger wear fault and the swash plate wear fault are very similar. Furthermore, as mentioned in reference [21], the spectral analysis approach is quite involved, and requires human experience in spectral analysis. The measurement technique itself requires attention to frequency ranges, resolution, filtering, windowing and averaging, particularly. 

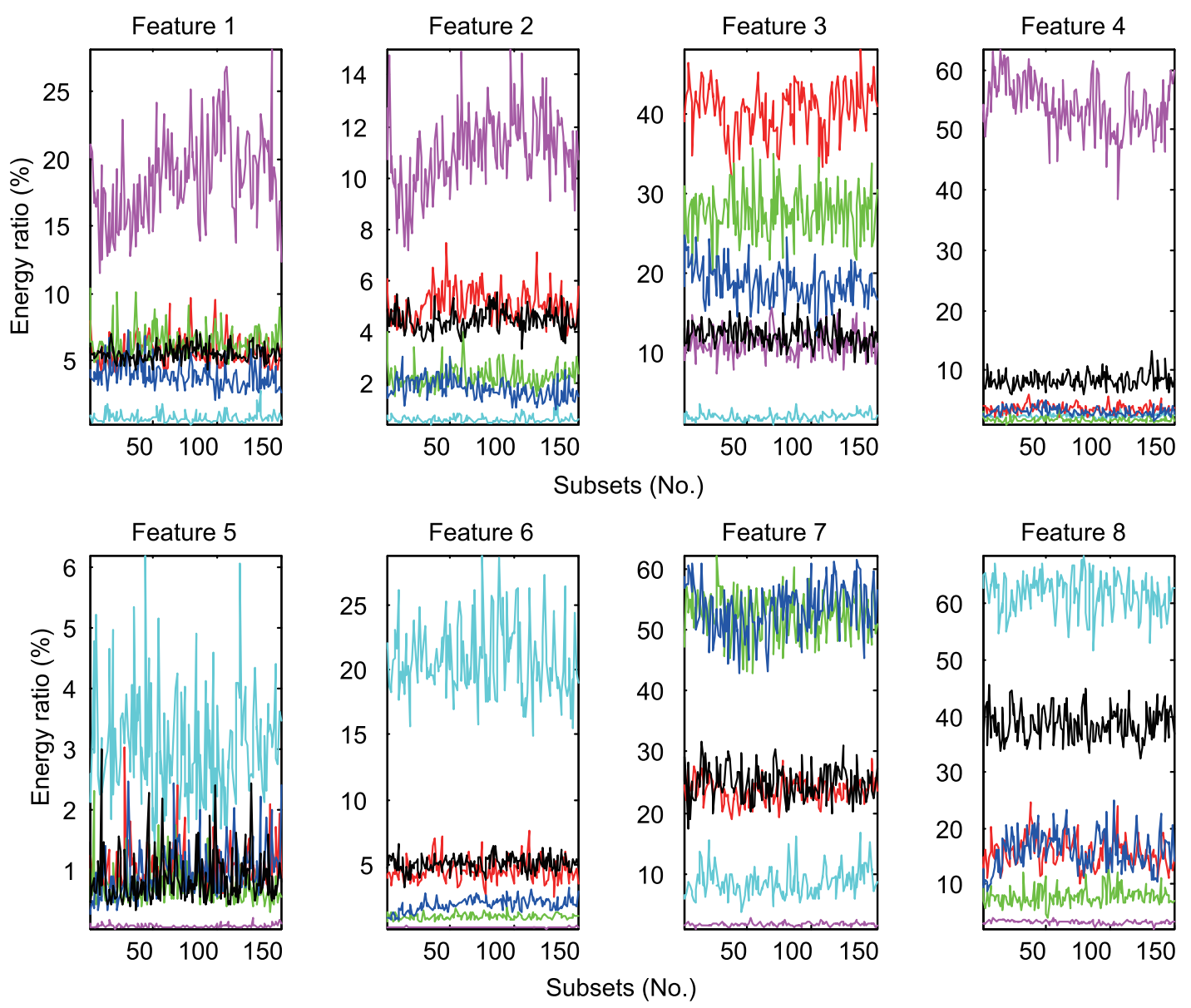

Fig. 8. Distribution of the features for the six types of training samples. The magenta line stands for norm state; the cyan line stands for bearing inner race fault; the red line stands for bearing roller fault; the green line stands for plunger wear fault; the blue line stands for swash plate wear fault; the black line stands for thrust plate wear fault.

Due to the good localization and multi-resolution features in the time-frequency domain, the wavelet transform has been widely used in the vibration analysis, and the wavelet packet energy feature has been adopted as an effective condition signature in the mechanical intelligent diagnosis method. Furthermore, as the industry progresses, there is less personnel and time available for condition monitoring. There is a demand for more automotive and supportive mechanism monitoring technology. In the next section, we utilize the PSO-RVM approach to implement the fault diagnosis, which is a machine learning based method.

\subsection{Fault diagnosis with PSO-RVMs and result analysis}

Each data set is divided into 190 segments, and each segment includes 1024 points. For each data set, select 150 subsets as training samples, and the other 40 subsets as testing samples. The waveforms of the six states are shown in Fig. 6. Every segment is decomposed into eight bins of wavelet packet coefficients with the 'daubchies 5' (Db5) wavelet at level 3. The 8 wavelet packet energy ratios are extracted as characteristic features, and shown in Fig. 7. It can be seen that several states are difficult to discriminate, especially, for the plunger wear fault and the thrust plate wear fault. Figures 8 and 9 are the distribution and the box-plot of the features for the six types training samples, respectively. From these two figures, because of the overlap and the intercross among features, it is extremely difficult to recognize all the six different conditions. So, it is necessary to adopt a more efficient intelligent classification method. 
Table 1

The obtained optimal parameters of RVM classifiers estimated by PSO

\begin{tabular}{lc}
\hline Model & Optimal value of $\gamma$ parameters \\
\hline PSO-RVM1 & 6.9370 \\
PSO-RVM2 & 8.1509 \\
PSO-RVM3 & 8.2844 \\
PSO-RVM4 & 4.8809 \\
PSO-RVM5 & 8.3039 \\
\hline
\end{tabular}

Table 2

Comparison of classification accuracies between RVMs with a given $\gamma=0.5$ and PSORVMs classifiers

\begin{tabular}{lcclcc}
\hline Model & $\begin{array}{c}\text { For training } \\
\text { samples/\% }\end{array}$ & $\begin{array}{c}\text { For testing } \\
\text { samples/\% }\end{array}$ & & $\begin{array}{c}\text { For training } \\
\text { samples/\% }\end{array}$ & $\begin{array}{c}\text { For testing } \\
\text { samples/\% }\end{array}$ \\
\hline PSO-RVM1 & 100 & 100 & RVM1 & 98.67 & 96.67 \\
PSO-RVM2 & 100 & 100 & RVM2 & 94.80 & 94.50 \\
PSO-RVM3 & 100 & 100 & RVM3 & 93.00 & 91.87 \\
PSO-RVM4 & 100 & 98.33 & RVM4 & 91.33 & 87.50 \\
PSO-RVM5 & 100 & 100 & RVM5 & 74.00 & 72.50 \\
\hline
\end{tabular}

Table 4

Comparison of the number of relevance vectors and support vectors for PSO-RVM and PSO-SVM, respectively

\begin{tabular}{lrrrrr}
\hline Model & 1st & 2nd & 3rd & 4th & 5th \\
\hline PSO-RVM & 6 & 4 & 3 & 6 & 2 \\
PSO-SVM & 46 & 46 & 30 & 17 & 14 \\
\hline
\end{tabular}

Comparison of diagnostic results between BP-NN, ANT-NN, PSOSVM, RVM, and PSO-RVM

\begin{tabular}{lc}
\hline Model & Diagnosis accuracies/\% \\
\hline BP-NN & 79.17 \\
ANT-NN & 83.33 \\
PSO-SVM & 99.58 \\
RVM & 71.25 \\
PSO-RVM & 99.17 \\
\hline
\end{tabular}

Feature 1

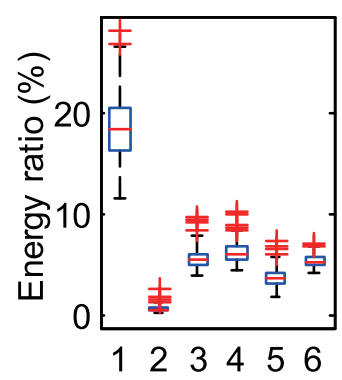

Feature 5

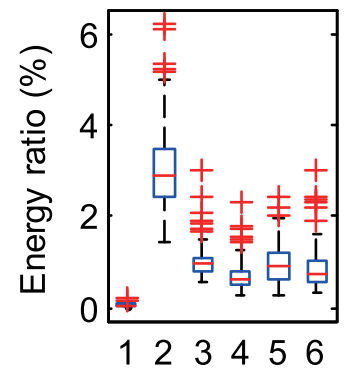

Feature 2

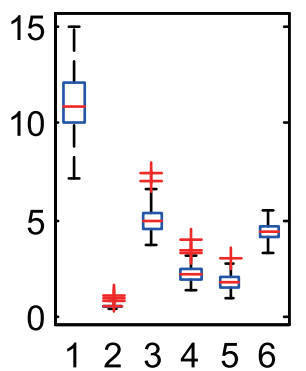

Feature 3

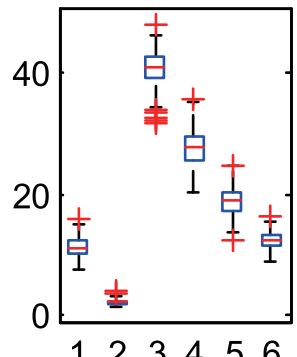

Feature 7

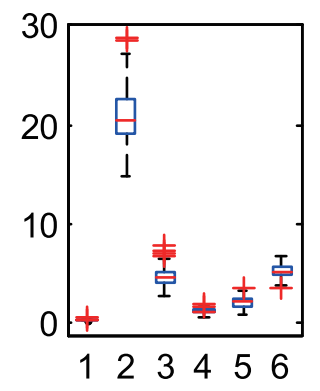

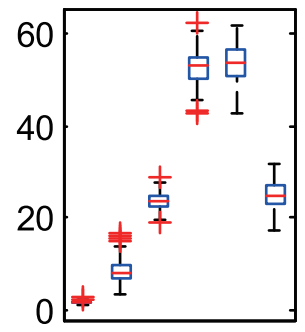

123456
Feature 4

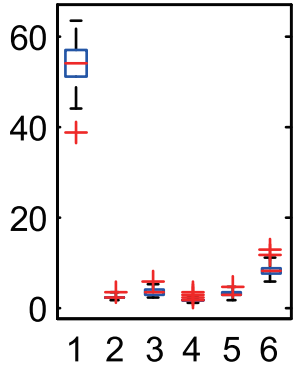

Feature 8

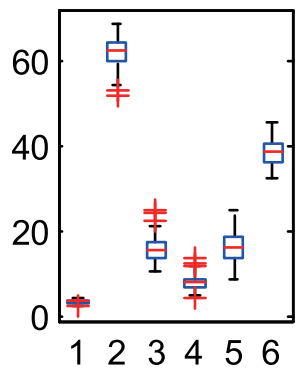

Types (No.)

Fig. 9. Box-plot of the features for the six types of training samples. The number in the $x$ axis stands for norm state, bearing inner race fault, bearing roller fault, plunger wear fault, swash plate wear fault, thrust plate wear fault, respectively.

All of the five RVMs adopt RBF as their kernel function. The PSO method is utilized to optimize the kernel width parameter $\gamma$. For each PSO-RVM, the adjusted parameter with maximal classification accuracy is selected as the most appropriate parameter with two-fold cross validation approach. Then, all of the training samples are trained to get the diagnosis model. The optimal parameters of RVM classifiers are listed in Table 1.

The first ANN compared with the proposed PSO-RVM is one hidden-layer BP-ANN with eight input nodes, eight hidden nodes and six output nodes. The second ANN compared with PSO-RVM is an ANT-ANN. The structure of 
the net is same to the mentioned BP-ANN, and the net weights are optimized by the ant colony algorithm [22]. The RVM compared with the proposed PSO-RVM is a general RVM with the kernel width 0.5. Furthermore, the PSORVM is compared with the recent PSO-SVM presented in the literature [18]. The training accuracy and the testing accuracy of each two-class classifier of the RVM and PSO-RVM are listed in Table 2. The classification performance comparison of BP-NN, ANT-NN, PSO-SVM, RVM, and PSO-RVM is presented in Table 3. The number of support vectors of PSO-SVM and that of relevance vectors of PSO-RVM are given in Table 4.

As shown in Table 3, the classification accuracies of PSO-RVM and PSO-SVM are obviously higher than those of BP-NN and ANT-NN, and the testing accuracy of BP-NN is only 79.17\%. This may attribute to that the BP optimization method is a local optimization method, whose ability of global search is very limited. In addition, the ANT colony enhances the performance of BP algorithm, but the classification accuracy is not very satisfying, only $83.33 \%$. Furthermore, without optimizing the kernel width parameter of the general RVM model, the performance is far from acceptable, and the accuracy is only $71.25 \%$. On the contrary, every two-class PSO-RVM gets a promising classification accuracy. As shown in Table 2, for PSO-RVM1, PSO-RVM2, PSO-RVM3, and PSO-RVM5, they classify correctly for all training and testing samples, and even for PSO-RVM4, there are only 2 misclassifications for the testing samples, and the classification accuracy reaches $98.33 \%$. It is noted from Table 3, PSO-RVM and PSO-SVM utilizing the global optimization of PSO, and combining with the powerful study capability of RVM and SVM, a very encouraging classification accuracy of $99.17 \%$ and $99.58 \%$ is achieved, respectively. As shown in Table 4, on the premise of no reduction of classification accuracy, the number of relevance vectors is far fewer than that of support vectors, which makes the diagnosis model much sparser and more appropriate for real-time diagnosis.

\section{Conclusion}

A novel PSO-RVM diagnostic method based on relevance vector machine with particle swarm optimization algorithm for plunger pump in truck crane is presented. In the model, the input is the vibration signal, and the output is the pump state. The PSO algorithm is employed to determine a suitable kernel width parameter of RVM classifier, which avoids over-fitting or under-fitting of the RVM model occurring. The real data from the plunger pump is used to verify the performance of the proposed PSO-RVM model. The experimental results show that the classification accuracy of PSO-RVM is superior to those of BP-ANN, ANT-ANN, and general RVM. The experimental result also indicates that the classification performance of PSO-RVM is competitive to that of PSO-SVM, but the number of relevance vectors is far fewer than that of support vectors of SVM. Therefore, the PSO-RVM model has more excellent performance for on-line real-time application.

\section{Acknowledgments}

This work is supported by the National Nature Science Foundation of China (Grant No. 61175038, 51205371), National Basic Research Program of China (“973” Program) (Grant No. 2013CB035400), Innovation Program of Shanghai Committee of Science and Technology (Grant No.11dz1121500, 11JC1405800), and also supported by the Research Project of State Key Laboratory of Mechanical System and Vibration (Grant No. MSV-2012-06).

\section{References}

[1] H. Chen, P.S.K. Chua and G. Lim, Adaptive wavelet transform for vibration signal modelling and application in fault diagnosis of water hydraulic motor, Mechanical Systems and Signal Processing 20(8) (2006), 2022-2045.

[2] Q. Wang, H. Chen, P. Chua and L.G. Hian, Fault detection of water hydraulic motor by demodulated vibration signal analysis with the hilbert transform and genetic algorithm, Journal of Testing and Evaluation 39(2) (2011), 12-15.

[3] Z. Zhao, M. Jia, F. Wang and S. Wang, Intermittent chaos and sliding window symbol sequence statistics-based early fault diagnosis for hydraulic pump on hydraulic tube tester, Mechanical Systems and Signal Processing 23(5) (2009), 1573-1585.

[4] K. Mollazade, H. Ahmadi, M. Omid and R. Alimardani, An intelligent model based on data mining and fuzzy logic for fault diagnosis of external gear hydraulic pumps, Insight: Non-Destructive Testing and Condition Monitoring 51(11) (2009), 594-600. 
[5] H.X. Chen, P.S.K. Chua and G.H. Lim, Fault degradation assessment of water hydraulic motor by impulse vibration signal with wavelet packet analysis and Kolmogorov-Smirnov test, Mechanical Systems and Signal Processing 22(7) (2008), 1670-1684.

[6] J.D. Wu, P.H. Chiang, Y.W. Chang and Y. Shiao, An expert system for fault diagnosis in internal combustion engines using probability neural network, Expert Systems with Applications 34(4) (2008), 2704-2713.

[7] A. EI-Shafei, T.A.F. Hassan, A.K. Soliman and Y. Zeyada, Neural network and fuzzy logic diagnostics of $1 \times$ faults in rotating machinery, Journal of Engineering for Gas Turbines and Power 129(3) (2007), 703-710.

[8] L. Guo, J. Chen and X. Li, Rolling bearing fault classification based on envelope spectrum and support vector machine, JVC/Journal of Vibration and Control 15(9) (2009), 1349-1363.

[9] J. Yang, Y. Zhang and Y. Zhu, Intelligent fault diagnosis of rolling element bearing based on SVMs and fractal dimension, Mechanical Systems and Signal Processing 21(5) (2007), 2012-2024.

[10] M.E. Tipping, Sparse bayesian learning and the relevance vector machine, Journal of Machine Learning Research 1(3) (2001), $211-244$.

[11] A. Widodo, E.Y. Kim, J.D. Son, B.S. Yang, A.C.C. Tan, D.S. Gu, B.K. Choi and J. Mathew, Fault diagnosis of low speed bearing based on relevance vector machine and support vector machine, Expert Systems with Applications 36(3 PART 2) (2009), $7252-7261$.

[12] C. He, C. Liu, Y. Li and J. Yuan, Intelligent fault diagnosis of rotating machinery based on multiple relevance vector machines with variance radial basis function kernel, Proceedings of the Institution of Mechanical Engineers, Part C: Journal of Mechanical Engineering Science 225(7) (2011), 1718-1729.

[13] X. Wang, M. Ye and C. Duanmu, Classification of data from electronic nose using relevance vector machines, Sensors and Actuators B: Chemical 140(1) (2009), 143-148.

[14] F. De Martino, A.W. de Borst, G. Valente, R. Goebel and E. Formisano, Predicting EEG single trial responses with simultaneous fMRI and relevance vector machine regression, NeuroImage 56(2) (2011), 826-836.

[15] W. Caesarendra, A. Widodo and B.S. Yang, Application of relevance vector machine and logistic regression for machine degradation assessment, Mechanical Systems and Signal Processing 24(4) (2010), 1161-1171.

[16] W. Pedrycz, B. Park and N. Pizzi, Identifying core sets of discriminatory features using particle swarm optimization, Expert Systems with Applications 36(3) (2009), 4610-4616.

[17] M. Korürek and B. Dogan, ECG beat classification using particle swarm optimization and radial basis function neural network, Expert Systems with Applications 37(12) (2010), 7563-7569.

[18] S. Fei, Diagnostic study on arrhythmia cordis based on particle swarm optimization-based support vector machine, Expert Systems with Applications 37(10) (2010), 6748-6752.

[19] W. Su, F. Wang, H. Zhu, Z. Zhang and Z. Guo, Rolling element bearing faults diagnosis based on optimal Morlet wavelet filter and autocorrelation enhancement, Mechanical Systems and Signal Processing 24(5) (2010), 1458-1472.

[20] Y. Ming, J. Chen and G. Dong, Weak fault feature extraction of rolling bearing based on cyclic Wiener filter and envelope spectrum, Mechanical Systems and Signal Processing 25(5) (2011), 1773-1785.

[21] A. El-Shafei and N. Rieger, Automated diagnostics of rotating machinery American society of mechanical engineers, International Gas Turbine Institute Turbo Expo (2003), 491-498.

[22] A. Kalinli, M.C. Acar and Z. Gündüz, New approaches to determine the ultimate bearing capacity of shallow foundations based on artificial neural networks and ant colony optimization, Engineering Geology 117(1-2) (2011), 29-38. 

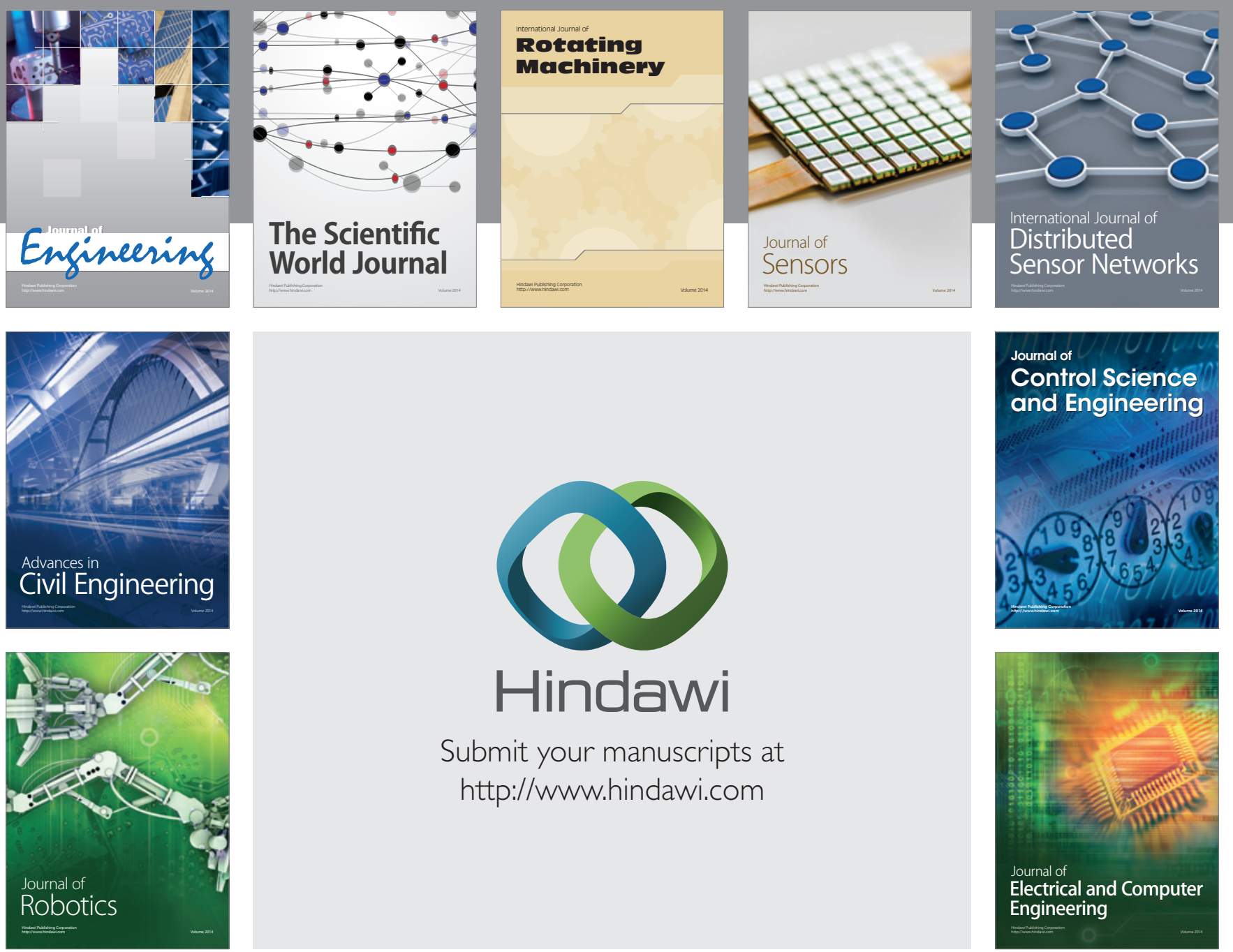

Submit your manuscripts at

http://www.hindawi.com
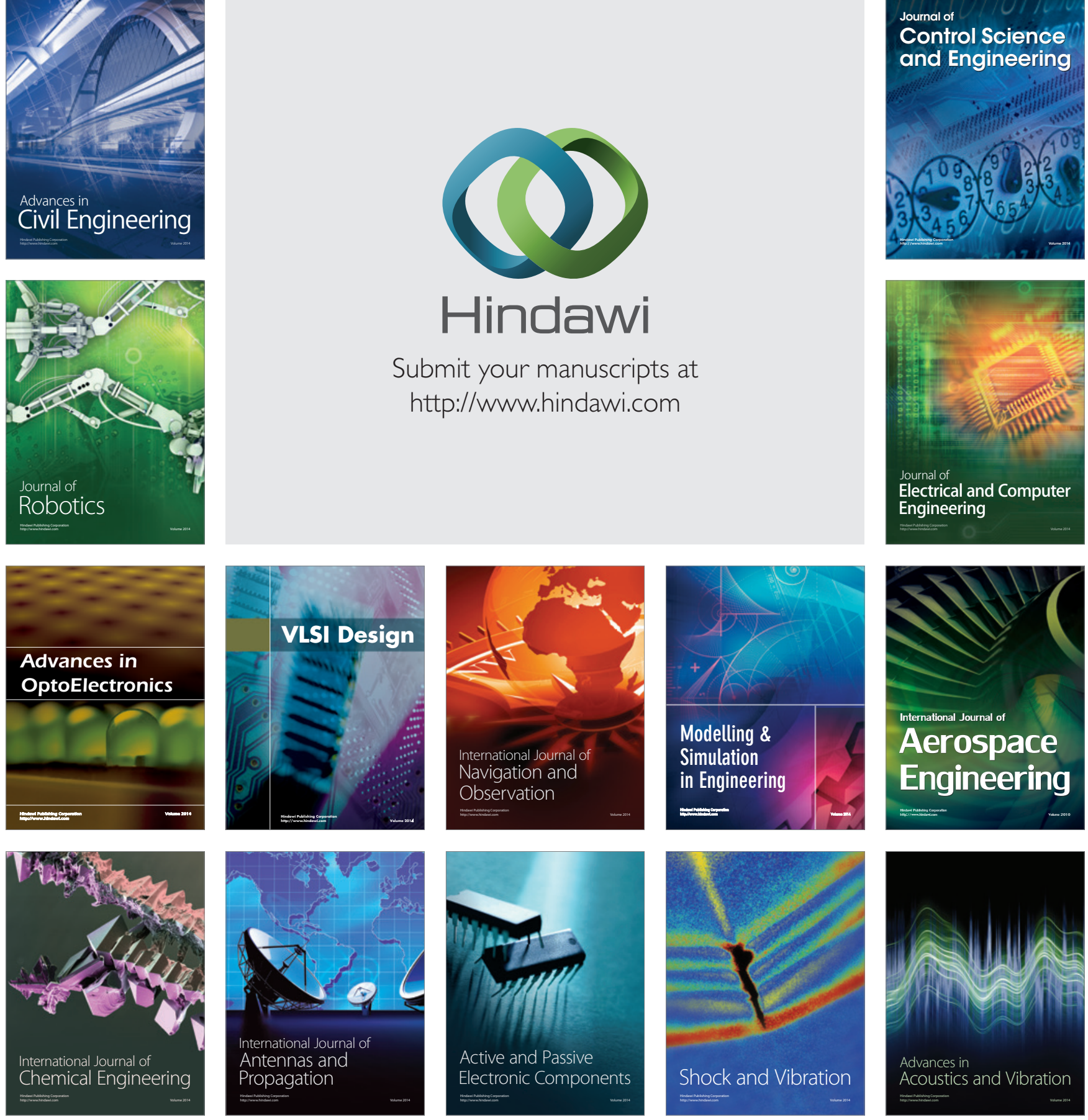